University of New Hampshire

University of New Hampshire Scholars' Repository

Physics Scholarship

Physics

4-1-2013

\title{
The radiation environment near the lunar surface: CRaTER observations and Geant4 simulations
}

M. D. Looper

J. E. Mazur

J. B. Blake

Harlan E. Spence

University of New Hampshire, harlan.spence@unh.edu

Nathan A. Schwadron

University of New Hampshire, Nathan.Schwadron@unh.edu

See next page for additional authors

Follow this and additional works at: https://scholars.unh.edu/physics_facpub

Part of the Physics Commons

\section{Recommended Citation}

Looper, M. D., J. E. Mazur, J. B. Blake, H. E. Spence, N. A. Schwadron, M. J. Golightly, A. W. Case, J. C. Kasper, and L. W. Townsend (2013), The radiation environment near the lunar surface: CRaTER observations and Geant4 simulations, Space Weather, 11, 142-152, doi:10.1002/swe.20034.

This Article is brought to you for free and open access by the Physics at University of New Hampshire Scholars' Repository. It has been accepted for inclusion in Physics Scholarship by an authorized administrator of University of New Hampshire Scholars' Repository. For more information, please contact Scholarly.Communication@unh.edu. 


\section{Authors}

M. D. Looper, J. E. Mazur, J. B. Blake, Harlan E. Spence, Nathan A. Schwadron, M. J. Golightly, A. W. Case, J. C. Kasper, and L. W. Townsend 


\title{
The radiation environment near the lunar surface: CRaTER observations and Geant4 simulations
}

\author{
M. D. Looper, ${ }^{1}$ J. E. Mazur, ${ }^{2}$ J. B. Blake, ${ }^{1}$ H. E. Spence, ${ }^{3}$ N. A. Schwadron, ${ }^{3}$ M. J. Golightly, ${ }^{3}$ \\ A. W. Case, ${ }^{4}$ J. C. Kasper, ${ }^{4}$ and L. W. Townsend ${ }^{5}$
}

Received 21 November 2012; revised 12 February 2013; accepted 24 February 2013; published 3 April 2013.

[1] At the start of the Lunar Reconnaissance Orbiter mission in 2009, its Cosmic Ray Telescope for the Effects of Radiation instrument measured the radiation environment near the Moon during the recent deep solar minimum, when galactic cosmic rays (GCRs) were at the highest level observed during the space age. We present observations that show the combined effects of GCR primaries, secondary particles ("albedo") created by the interaction of GCRs with the lunar surface, and the interactions of these particles in the shielding material overlying the silicon solid-state detectors of the Cosmic Ray Telescope for the Effects of Radiation. We use Geant4 to model the energy and angular distribution of the albedo particles, and to model the response of the sensor to the various particle species reaching the 50 kilometer altitude of the Lunar Reconnaissance Orbiter. Using simulations to gain insight into the observations, we are able to present preliminary energy-deposit spectra for evaluation of the radiation environment's effects on other sensitive materials, whether biological or electronic, that would be exposed to a similar near-lunar environment.

Citation: Looper, M. D., J. E. Mazur, J. B. Blake, H. E. Spence, N. A. Schwadron, M. J. Golightly, A. W. Case, J. C. Kasper, and L. W. Townsend (2013), The radiation environment near the lunar surface: CRaTER observations and Geant4 simulations, Space Weather, 11, 142-152, doi:10.1002/swe.20034.

\section{Introduction}

[2] CRaTER, the Cosmic Ray Telescope for the Effects of Radiation, has been in orbit around Earth's moon aboard the Lunar Reconnaissance Orbiter (LRO) since June 2009. The sensor is designed to measure the radiation environment near the Moon, with emphasis on the radiation dose that will be absorbed by sensitive materials, whether electronic parts or living tissue, behind shielding. In particular, the silicon solid-state detector stack incorporates two large cylindrical pieces of A-150 tissue-equivalent plastic

\footnotetext{
${ }^{1}$ The Aerospace Corporation, El Segundo, California, USA.

${ }^{2}$ The Aerospace Corporation, Chantilly, Virginia, USA.

${ }^{3}$ Institute for the Study of Earth, Oceans, and Space, University of New Hampshire, Durham, New Hampshire, USA.

${ }^{4}$ Harvard-Smithsonian Center for Astrophysics, Cambridge, Massachusetts, USA.

${ }^{5}$ Nuclear Engineering Department, University of Tennessee, Knoxville, Tennessee, USA.
}

Corresponding author: M. D. Looper, The Aerospace Corporation, El Segundo, California, USA. (mark.d.looper@aero.org)

(02013. American Geophysical Union. All Rights Reserved. 1542-7390/13/10.1002/swe.20034
(TEP), representing the muscle tissue overlying an astronaut's radiation-sensitive bone marrow [Spence et al., 2010]. The detecting elements are made of silicon, and the effects of TEP and other shielding can be related to those of an equivalent mass of, e.g., aluminum, so that the CRaTER observations are relevant to the radiation exposure of space electronics and to that of astronauts.

[3] Figure 1 shows a diagram of the sensor geometry, generated by the Geant 4 code discussed in section 3 below; details of the instrument are given by Spence et al. [2010]. The detector stack of CRaTER consists of six silicon solid-state detectors in three pairs. Each pair consists of one thin and one thick detector, nominally 148 microns and 1 millimeter thick, respectively, and neighboring pairs are separated by one of the two pieces of TEP mentioned above. The electronics for the thin detectors have higher thresholds and a higher energy deposit saturation level than those for the thick detectors, so that the thin detectors are insensitive to protons and most alphas but can measure the greater energy deposits from heavy ions, while the thick-detector pulse-height analysis measures energy deposits down to below the minimum ionization level of a singly charged particle (about $0.3 \mathrm{keV}$ per micron in silicon), but saturates if hit by an energetic ion of more than moderately high nuclear charge $Z$. The detectors are 


\section{LOOPER ET AL.: LUNAR RADIATION ENVIRONMENT: CRATER}

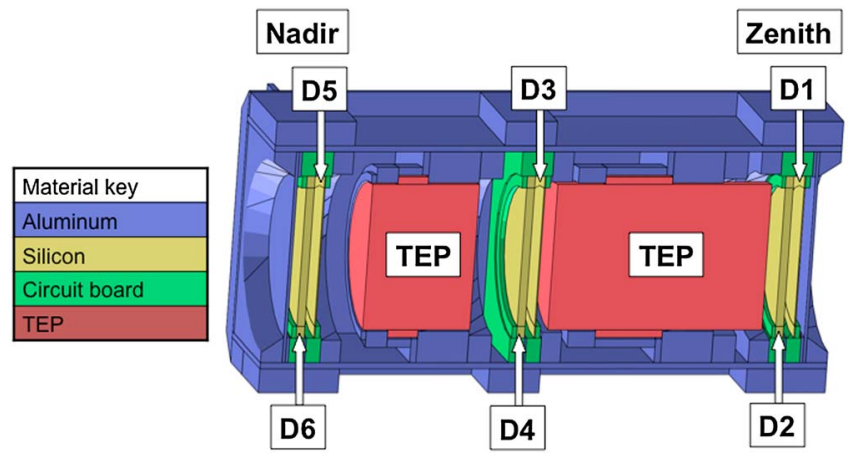

Figure 1. Image of the sensor-head simulation geometry generated by the Geant 4 code. Silicon detectors (yellow) are D1 to D6 from right to left, with odd-numbered detectors 148 microns thick and even-numbered detectors 1 millimeter thick. Blue is aluminum, green is printedcircuit-board material in the detector mounts, and red is A-150 tissue-equivalent plastic (TEP). In the nominal orientation of LRO, the sensor axis is straight up and down, with D1 toward the zenith and D6 toward the lunar surface.

numbered D1 through D6 from the right side of Figure 1 to the left, with thin detectors having odd numbers and thick detectors having even numbers. In the nominal mapping attitude of LRO, the sensor axis points straight up and down, with D1 toward space and D6 toward the Moon.

[4] CRaTER is different from most cosmic-ray telescope instruments in that its scientific focus is not on determining the primary particles entering the sensor (although it can do this to some degree, as shown below). Instead, it was designed primarily to study the effects of radiation that would be experienced by astronauts or equipment at and near the Moon: the energy deposited in the sensitive materials, whether biological or electronic, that these particles strike [Spence et al., 2010]. The inclusion of inert material (the TEP) in the detector stack is not advantageous for determining the species and energy spectra of particles incident on the sensor; rather, its purpose is to enable study of the effects of the nuclear and electromagnetic interactions of those particles as they pass through this overlying material on their way to a sensitive target.

[5] This means that the measurements of CRaTER are particular to the configuration of the sensor: instead of using sensor-specific measurements to extract energy spectra of particles in free space outside the sensor, we report below mostly the energy-deposit spectra in the detectors of CRaTER, surrounded by their particular arrangement of shielding material. One way to abstract energy-deposit measurements from the geometric details of a particular sensor is to normalize energy deposit to the particles' pathlength through the detector volumes; this is commonly referred to as linear energy transfer, or LET. CRaTER telemetry includes measurements of the energy deposits in the individual detectors, with information about which detectors were triggered in coincidence with one another. By restricting consideration to events triggering, say, the three thick detectors all together, we can define a collimated set of events entering the sensor near the axis and passing through a well-defined stack of material thicknesses. Thus, for example, cosmic rays entering the D1 end of the sensor and traversing all three thick detectors will pass through 9.9 grams per square centimeter of material to reach D6, so one could divide the energy deposits in D6 by its thickness to report the LET spectrum under 9.9 grams per square centimeter, which would be representative of the spectrum under the same column mass density of any material. If the thickness of the detector is reported as a column density, so that the LET is measured in, e.g., kiloelectron-volts per (grams per square centimeter), then again the result would be applicable to any sensitive target material.

[6] The purpose of this paper is to show the origins and relative magnitudes of various contributions (galactic cosmic rays (GCR), secondary species, and background) to the energy-deposit event distributions observed by CRaTER in lunar orbit, and to use this information to derive a preliminary LET spectrum, as described above, from the observations. We expect our results to improve in future work with additions to our Geant4 modeling, as will be discussed below. Other members of the CRaTER team present LET spectra and related analyses in this issue using different techniques (A. W. Case et al., The DeepSpace Galactic Cosmic Ray Lineal Energy Spectrum at Solar Minimum, submitted to Space Weather, 2013; J. A. Porter et al., Analyses of Primary and Secondary Ion Contributions to LET Spectra for the CRaTER Instrument on LRO, submitted to Space Weather, 2013; C. J. Zeitlin et al., Measurements of Galactic Cosmic Ray Shielding with the CRaTER Instrument, submitted to Space Weather, 2013).

[7] Actually, the term LET is not quite correct for the CRaTER measurements reported here, although its usage in this context is commonplace. According to the definitions of the International Commission on Radiological Protection [ICRP, 1991], LET properly refers to the energy lost by the particle in traversing a given thickness of material. However, unless all of the lost energy is actually deposited in the material, with none escaping as energetic secondary particles that leave the measurement volume, then the quantity actually measured by detectors like those in CRaTER is the energy absorbed by the material. Dividing the average energy deposit in a target by the mean chord length of particles traversing it yields a quantity called lineal energy; if the target is thick enough to capture any secondary particles, but thin enough that primary particles' LET does not change appreciably due to energy loss as they traverse it, then lineal energy will be numerically equal to the average LET of the particles. We will discuss the distinction between LET and lineal energy more in section 4 , and in the meantime, we will report observations simply as the energy deposit divided by the detector thickness ("normalized energy deposit"), which is a stand-in for both these quantities and has the same units, here kiloelectron-volts per micron (of silicon). 


\section{Observations}

[8] In this work, we will focus on a homogeneous sample of CRaTER data from early in the LRO mission, at the end of the period of record-high GCR flux reaching Earth during the recent deep and prolonged solar minimum [Mewaldt et al., 2010]. The period we will analyze begins when LRO entered its mapping orbit, nominally circular at 50 kilometers altitude, on day 259 of 2009 , and ends on day 65 of 2010 before the first Forbush decrease of the mission. During this time the GCR flux was high and steady, and there were no solar particle events at energies relevant to CRaTER observations. CRaTER operations were likewise constant, with no instrument mode changes.

[9] Data from six days on which in-flight calibrations occurred were cut from the sample and another six days were lost to telemetry gaps, leaving a total of 160 days' data. While in the mapping orbit, LRO is nominally fixed in orientation with its imaging instruments pointing at the nadir; we cut from our sample the small fraction of time when this attitude was not maintained within one degree. The result of these selection criteria is a sample of the near-lunar radiation environment with steady environmental conditions and essentially constant observational parameters. The LRO altitude actually varied from about 35 to 70 kilometers during this period, although the mean altitude of each orbit was steady. However, the simulations discussed below do not show substantial deviation of expected instrument response to the radiation environment over this range. Thus, we ignore altitude variations in the period of these observations, and all simulations in this work are performed with the sensor at the nominal altitude of 50 kilometers. The result is an operationally consistent data set, which we used to observe and model the lunar radiation environment during the recent solar-minimum period of record-setting GCR flux.

[10] Figure 2 shows a subset of the observations accumulated during this period, to illustrate some of the main contributors to the energy-deposit spectra observed in single detectors. This is a plot of energy deposits in thick detectors D4 and D6, which are in the thin/thick detector pairs closest to the nadir-facing end of the sensor, for particle events triggering those two detectors but not D2 at the zenith-facing end (thin detectors are ignored in this sample). The thick detectors saturate at an energy deposit of about 90 kiloelectron-volts per micron and the thin detectors at about 2000 kiloelectron-volts per micron, so this plot focuses on the lower end of the CRaTER energy deposit range.

[11] The plot is dominated by GCRs, which for this sample come into the detector stack through the wall between the D3/D4 and D1/D2 pairs (so as not to trigger D2), and traverse first D4 and then D6. Because the LET of energetic ions increases with decreasing energy, and because a GCR ion will lose energy traversing the TEP between D4 and D6, we expect GCR protons to show up as a track on this plot with D4 energy deposit lower than that in D6, i.e., below the diagonal where both energy deposits would be

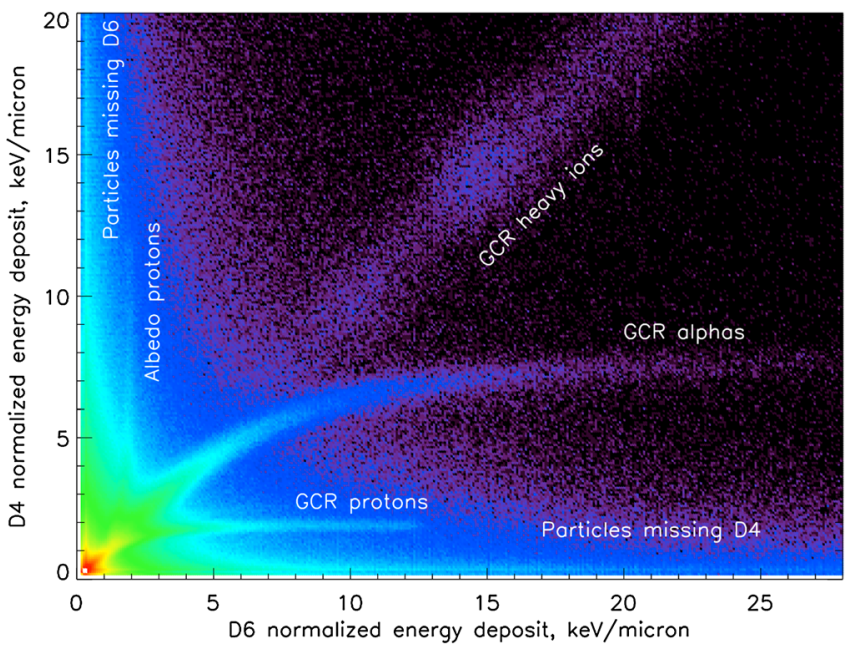

Figure 2. Distribution of all particle events observed during the period of the study that had detectors D4 and D6, but not D2, triggered. The six-decade logarithmic color scale indicates density of points per pixel per unit time in plot of events' energy deposit in D4 vs. that in D6. Tracks due to GCR protons and alphas coming from above and for protons coming up from the lunar surface, and a train of peaks along the diagonal due to relativistic heavier GCR ions, are labeled. "Wings" due to particles striking one detector and missing the other, but triggering it remotely via a delta ray, are also labeled.

equal. Higher-energy GCR protons lose a smaller fraction of their energy in traveling from D4 to D6, so the portions of the track with lower energy deposits (due to these higher-energy protons) will be closer to the diagonal. Finally, a curve of LET vs. energy flattens out at 0.3 kiloelectron-volts per micron, the so-called "minimum ionizing" value, for singly-charged particles at relativistic energies (about 1 gigaelectron-volt for protons). Because the GCR proton spectrum contains many such particles, we expect the track to intensify as many protons stack up in a spot with both D4 and D6 energy deposits near this value. A curve having exactly these characteristics is clearly visible in the plot, labeled as "GCR protons." Because the energy deposit of ions at a given velocity is approximately proportional to $Z^{2}$, we expect GCR alphas to produce a track similar to that from protons but scaled in both axes by a factor of 4 , and such a track is likewise visible and labeled in the plot. Less abundant heavy ions among the GCR produce fainter tracks, so that most of what we see is the relatively intense cluster of "hits" due to the relativistic part of their spectra at an energy deposit of about 0.3 kiloelectron-volts per micron times $Z^{2}$ in both D4 and D6. A diagonal line of such "clouds" due to GCRs heavier than alphas is labeled in Figure 2 (notable are carbon around 10 kiloelectron-volts per micron and nitrogen around 15 kiloelectron-volts per micron).

[12] Two other populations stand out in Figure 2. First, protons coming up from the Moon with at least 
60 megaelectron-volts of energy will traverse first the nadir-facing D6 and then D4, losing energy in the TEP between them, so they should produce a curve that is a mirror image across Figure 2's diagonal of the GCR proton curve. A track due to such "albedo protons", which are secondary particles resulting from the interaction of GCR primaries with the lunar surface, is labeled in Figure 2. We have reported on some characteristics of this population, including their global distribution over the lunar surface [Wilson et al., 2012]. Second, there are unstructured "wings" close to and paralleling both axes, with energy deposit in one detector concentrated around the minimum ionizing value for singly charged particles of 0.3 kiloelectron-volts per micron and with higher energy deposit in the other detector, and with greater intensity toward the origin of the plot. Our instrument and albedo simulations, as described in section 3 below, show that some of these events with lower energy deposits are due to albedo electrons and positrons, with a small fraction due to albedo neutrons and gammas (to which CRaTER is quite insensitive). However, most of them, especially farther from the origin along each axis, are due to GCR ions striking the detector that shows the greater energy deposit and missing the other, but triggering it remotely via a secondary particle (usually a knock-on electron, also known as a delta ray) that travels away from the ion's trajectory and deposits a small amount of energy in the detector missed by the ion to complete the coincidence. These "wings," with fringes partially overlapping the GCR and albedo proton tracks, are labeled in the plot according to which detector the causative primary particles missed.

[13] Support for this interpretation of the events in the "wings" is provided in Figure 3, which plots energydeposit spectra in adjacent thin and thick detectors D1 and D2 for several classes of particle events. Because these detectors are right next to each other, one might expect that a particle crossing one would be very likely to cross the other and to have essentially the same energy loss per unit pathlength in both. This expectation is borne out by the upper and lower pairs of spectra in Figure 3: the upper pair of curves represents the energy deposits of all events triggering the respective detector without requiring any other detector to be either triggered or not triggered, while the bottom pair of curves is for energy deposits of events triggering all six detectors. In the overlap between threshold and saturation for any of the detectors, about 3 to 90 kiloelectron-volts per micron, the two curves in each of these pairs are indeed very close to one another (some systematic differences between the two six-detector curves will be investigated in section 4).

[14] One might expect the curve for triple coincidences of the thick detectors (D2, D4, and D6, ignoring whether the thin detectors were triggered or not) to be similar to that for sixfold coincidences, above the threshold where the thin detectors are triggered. That is, if the rate of energy loss of a given particle in a thick detector is also high enough to trigger a thin detector, then it is very likely that it will trigger the adjacent thin detector, and so all triple coincidence events counted in that spectrum above

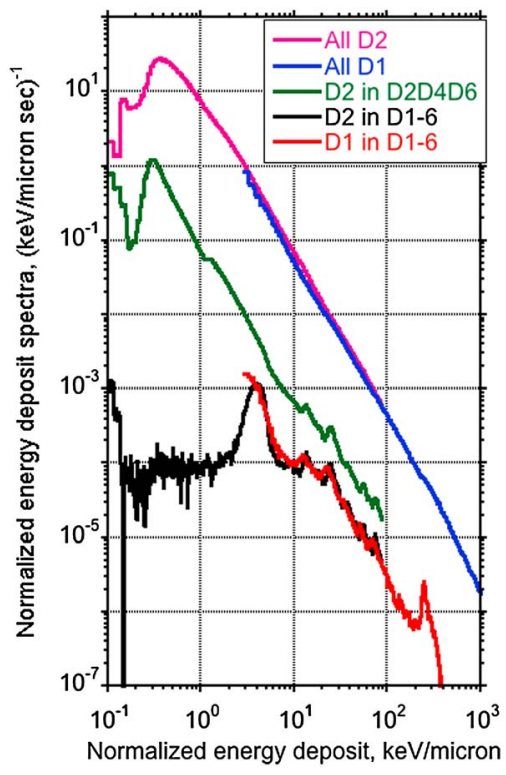

Figure 3. Energy-deposit spectra in the zenithward pair of detectors for different classes of events observed during the study period. The two highest curves are for all events triggering D2 or D1, respectively; the single curve in the middle is the spectrum of energy deposits in D2 for all events that trigger all three thick detectors; and the bottom two curves are the spectra in the two detectors for events triggering all six detectors.

about 3 kiloelectron-volts per micron should also be counted in the sixfold coincidence spectrum. However, the spectrum of energy deposits in D2 for these triple coincidence events in Figure 3 is about a factor of three above the curves for sixfold coincidences.

[15] Looking at plots like that in Figure 2 for energy deposits in pairs of thick detectors for triple-coincidence events (not shown, but very similar to Figure 2), one sees that these extra events are contributed by "wings" similar to those for the double-coincidence events in that Figure, where a particle traverses D2 and D4 (or D4 and D6) and misses D6 (D2) but triggers it remotely via a small energy deposit from a delta ray. Such an event, with a low energy deposit in one of the thick detectors, is counted as a triple coincidence, but the high threshold of the thin detectors would cause a similarly small energy deposit in the adjacent thin detector to fail to trigger it, so that the event would not be counted as a sixfold coincidence. Because this means that events coming from outside the nominal geometric acceptance cone defined by the D2, D4, and D6 detectors can be counted as triples, we cannot use the straight-lines geometric factor of $0.605 \mathrm{~cm}^{2} \mathrm{sr}$ to "divide out" the sensor's specific contribution to the measurement. In section 4, we will discuss how we can still abstract the measurement from the details of the sensor geometry in spite of this complication. 


\section{LOOPER ET AL.: LUNAR RADIATION ENVIRONMENT: CRATER}

\section{Simulations}

[16] To understand the details of the observations, and how to use them to extract observations about the radiation environment from observations specific to the sensor, we performed extensive simulations of the interactions of GCRs with the lunar surface and of the response of the CRaTER sensor to the particles striking it. We used Geant 4 (GEometry ANd Tracking), which is a Monte Carlo radiation transport code developed by the high-energy particle physics community but with an increasing user base among space scientists and engineers [Allison et al., 2006]. This code package simulates the trajectories of individual energetic particles of arbitrary species through arbitrary threedimensional material geometries, sampling from probability distributions of continuous processes (e.g., fluctuations in energy deposit) and discrete processes (e.g., nuclear interactions with the target material). The code package requires that the physics processes to be simulated be defined in a "physics list," so for the simulations in this work using version 9.3 of Geant 4 we selected a physics list based on the "radioprotection" example provided with the code package.

[17] Table 1 lists some of the specifics of the processes we activated in our simulations, both of the sensor response and of lunar secondary particle production from GCR impacts. The interactions and transport of electrons, positrons, photons, and hadronic particles and nuclei up to alphas are simulated in great detail; however, for the present work we only simulated electromagnetic processes (ionization energy loss, scattering, etc.) for nuclei heavier than alphas. This means that the observed broadening of energy-deposit peaks by fluctuations in energy deposit, for example, will be reproduced in the simulations, but that no nuclear interactions will fragment heavy-ion projectiles as they pass through the sensor. Other members of the CRaTER team have used other codes to simulate these nuclear interactions in the sensor head (Porter et al., submitted manuscript, 2013; Zeitlin et al., submitted manuscript, 2013), and we plan to add them to our future Geant 4 work, but some effects of this omission will be discussed in section 4. With regard to the effects of omitting these processes from the simulation of the lunar albedo production, we have made some preliminary calculations to help choose a physics list (under Geant4 version 9.6) with nuclear interactions of heavy-ion projectiles. The results suggest that, given the specifics of the observations being modeled (energy threshold of $\sim 60$ megaelectron-volts for albedo protons to trigger coincidence measurements, sensor looking straight down at the lunar surface, very little sensitivity to neutrons), we have captured most of the albedo particle production relevant to this data set. However, we also intend in future work to look at data taken during occasions when CRaTER is pointed toward the lunar limb, and fragments coming from heavy GCR ions after a glancing impact on the lunar surface will likely make a greater contribution to those measurements.

[18] As shown in Figure 2, we see clear indications in the CRaTER observations of particles coming up from the Moon. These are secondary particles produced by the interactions of GCRs with the lunar surface, and as is conventional we refer to them by the somewhat imprecise term "albedo" (some object to the use of this term to label secondary particle populations that are not simply reflected primary particles). To simulate these, we illuminated a point at the center of one side of a slab of material isotropically with GCR protons and alphas, and tabulated all particles that returned upward through that side and also all particles that reached the nominal 50 kilometer altitude of LRO. (Our 50 kilometer "counting surface" was a sphere 1788 kilometers in radius centered 1738 kilometers below the target point, so that the greater distance traveled by particles coming off the surface at oblique angles instead of straight up was properly accounted for. In particular, this is important for the decay in flight of unstable secondary particles like pions and muons.)

[19] Several models are available to calculate GCR primary spectra as input to our simulations; for a recent comparative review see Mrigakshi et al. [2012]. We chose to use the Badhwar-O'Neill model [O'Neill, 2010], which provides GCR fluxes for all elements up to nickel (with extrapolation for elements from copper up to plutonium, which we did not use), encapsulating the varying effects of heliospheric transport in a single "modulation parameter" $\Phi$ with dimensions of rigidity. O'Neill [2010] says that the parameter giving the best fit between the model and ACE oxygen observations during the deep solarminimum period in this study was " 430 megavolts"; for consistency with related calculations using a somewhat earlier version of the Badhwar-O'Neill model [e.g., Townsend et al., 2011], we used a slightly different modulation parameter of 417 megavolts.

[20] To simulate the lunar surface, we modeled a slab of ferroan anorthosite, with composition as given by Gasnault et al. [2000]. We tested a variety of targets representing typical lunar highland and lowland compositions, and saw only insignificant variations in the distributions of the output particles of species to which CRaTER is sensitive (the largest variations were in neutrons, but CRaTER

Table 1. Physics Processes Included in the Geant4 Simulations

\begin{tabular}{|c|c|}
\hline Physics Process & Comments \\
\hline Electromagnetic energy loss & $\begin{array}{l}\text { Includes scattering and } \mathrm{d} E / \mathrm{d} x \text { fluctuations for realistic straggling of range and } \\
\text { energy deposit }\end{array}$ \\
\hline Secondary particle production & $\begin{array}{l}\text { Includes creation of knock-on electrons (delta rays) and bremsstrahlung photons } \\
\text { that can carry energy away from detector volumes }\end{array}$ \\
\hline Nuclear interactions & Based on Geant4 Binary Cascade model; only applicable for protons and alphas \\
\hline
\end{tabular}


is quite insensitive to those, as seen below), so this choice of material is not critical for this work. More critical is to make sure that the target is thick enough to contain all incident and secondary particles, so that we do not miss whatever upward-escaping secondary particles might have been produced by any particles (primary or secondary) that escape out the back of a too-thin slab. Jia and Lin [2010] found a thickness of 10 meters with a loose regolith-like density of 2 grams per cubic centimeter to be sufficient. We simulated a slab 10 meters thick but with the 3 grams per cubic centimeter density of Gasnault et al. [2000], and gave it dimensions of 1 kilometer square in the horizontal plane.

[21] Assuming a uniform and isotropic GCR incidence over the lunar face visible to LRO, symmetry and Liouville's theorem allow us to map the angular distribution of particles reaching a sphere 50 kilometers above the simulated point target into an angular distribution for each species observed at LRO, with the sky that is not obscured by the lunar disk being filled by an isotropic primary GCR flux. (At 50 kilometer altitude, the Moon subtends an angle of about $153^{\circ}$.) Figure 4 shows the results of this simulation, aggregated as an integral of the fluxes of various species over all angles at LRO. Many muons, pions, and other unstable species come up from the surface, but by the time they reach 50 kilometers all of the muons and pions, and all but a small fraction of other species at the highest energies (i.e., those most

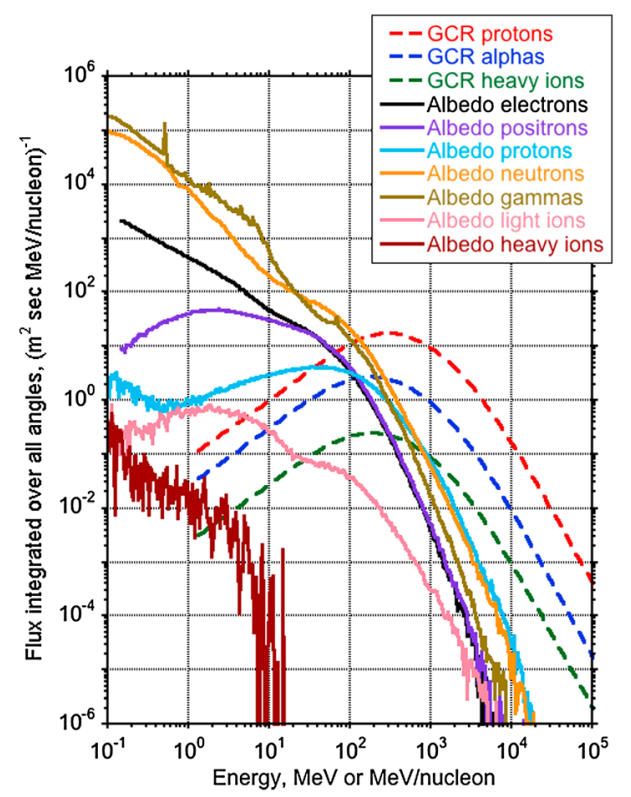

Figure 4. Energy spectra of primary GCR species (dashed lines) from the Badhwar-O'Neill 2010 model [O'Neill, 2010] model with modulation parameter $\Phi=$ $417 \mathrm{MV}$, and of lunar secondary ("albedo") species calculated with Geant4. Spectra are integrated over all angles for an observer at the nominal LRO altitude of 50 kilometers. affected by relativistic time dilation), have decayed to electrons and positrons (half to two thirds of the electrons and positrons above about 100 megaelectron-volts at 50 kilometers are from this source). "Albedo light ions" in the plot are hydrogen and helium isotopes other than protons, and "albedo heavy ions" are secondary nuclei heavier than helium; these are not significant contributors to the energy-deposit spectra observed by CRaTER, and are not discussed further. GCR fluxes are also shown as dashed lines for protons, alphas, and heavier ions, integrated over the sky not blocked by the Moon. The spectra of most secondary-particle species harden and intensify as one looks from the nadir toward the lunar limb. We do not discuss this further here, instead simply convolving the simulated energy and angular distributions of particles with the angular response of the sensor in its nominal zenith/nadir attitude, but in future work we intend to compare simulations with observations during periods when LRO points away from its nominal attitude. (As noted above, this is also where the addition to our simulation of nuclear fragmentation products from glancing impact of heavy GCR nuclei is likely to make a greater contribution.)

[22] The geometric model of the CRaTER sensor head that we used in simulating the instrument's response was shown above as Figure 1, in a cross-sectional diagram produced by the Geant 4 code itself. For the results reported here, we illuminated the sensor head from all angles with ions up to nickel and with the predominant albedo species from Figure 4 (photons, neutrons, electrons, and positrons) at energies covering the range in that Figure, and tabulated all energy deposits whether in single detectors or in multiple detectors simultaneously. From this we are able to calculate the response in single detectors and in multiple-detector coincidences to any of these species at any energy and arriving from any angle. We then convolved this "kernel" with the full energy and angular distribution of GCRs and secondary species, from the earlier simulation that was summarized in Figure 4.

[23] We also performed separate simulations with the detailed model of the sensor head attached to a similarly detailed model of its electronics box [see Spence et al., 2010], which in turn was attached to a simplified mass model of the entire LRO spacecraft. This geometry was illuminated by energetic particles as described above, in separate sets of runs with the spacecraft's hydrazine tanks filled and empty. We were concerned as to whether, for example, neutrons striking the large quantity of hydrazine in the tanks could liberate enough energetic protons to make a noticeable difference in the sensor's response. However, we found that except for a small fractional increase in protons due to GCR alphas breaking up in the material of the spacecraft, there was no discernible change in response in this much more cumbersome simulation, and so we have restricted consideration to the response of the "bare" sensor head as shown in Figure 1.

[24] As a sample of the results of this convolution of incident particles with sensor response, we show Figure 5. This is a simulation of the distribution of energy deposits 


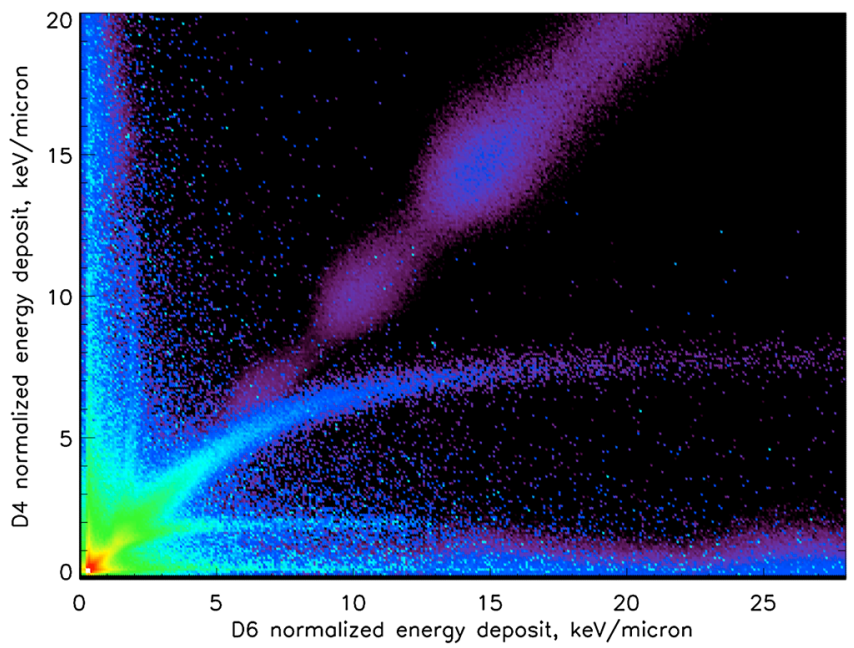

Figure 5. Distribution of simulated events in CRaTER triggering D4 and D6 but not D2. Colorscale is the same as that for Figure 2. Tracks labeled in that figure are all in the right places and at about the right intensities, as is the peak of minimum-ionizing particles depositing about 0.3 kiloelectron-volts per micron in each detector; diffuse "wings" of events paralleling the axes are not as strong as in the observations.

in detectors D4 and D6 for events triggering a double coincidence of these detectors but not D2 (and ignoring the state of the thin detector triggers). It is directly comparable with Figure 2; the six-decade logarithmic color scale is the same. As can be seen, the major features in the observations are all present in the simulations, and at about the right intensities, but one notable difference is that the "wings" paralleling the axes are not as strong and are more highly structured, showing lumps at energies corresponding to the "clouds" of relativistic heavy ions along the diagonal (which are likewise tighter than those in the observations). As a preliminary conclusion, we attribute this to our incomplete physics list, which omits fragmentation of GCR nuclei heavier than alphas and thus may be missing a variety of particles that could contribute to the "wings." We expect the simulation fidelity to improve in future work when we add nuclear interactions to our simulation of heavy ion projectiles.

[25] To quantify the contributions of various species and the comparison with observations, in Figures 6 and 7 we show energy-deposit spectra in detector D6. Figure 6 shows the spectra for all events triggering that detector without reference to the state of other detectors' triggers, and Figure 7 shows spectra for events producing threefold coincidences of the thick detectors D2, D4, and D6. Both plots show the contributions to the total energy-deposit spectrum from the various GCR and albedo constituents, although the contribution of photons and neutrons to the plot for three-detector coincidences is negligible. GCRs dominate the energy deposit in both figures, such that if we integrate over these curves to produce dose rates,

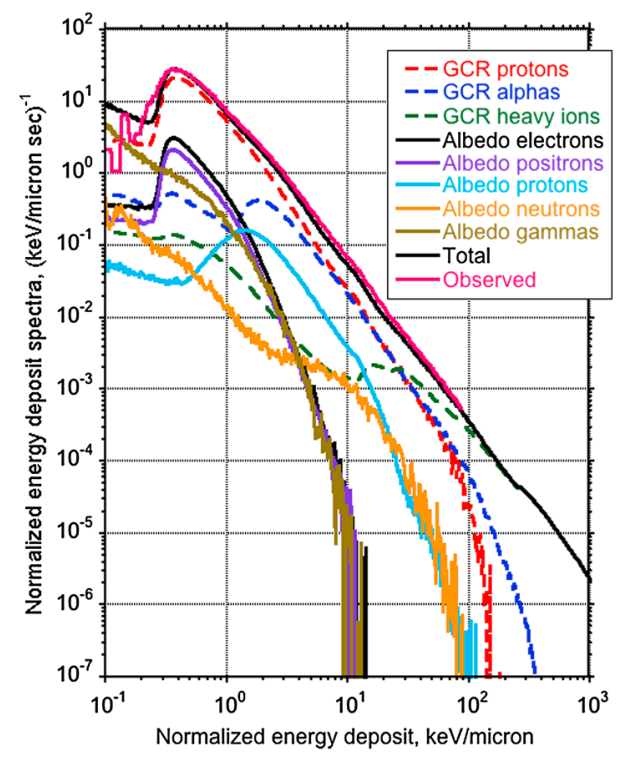

Figure 6. Energy deposit spectra in D6 of all events triggering that detector, comparing the observed spectrum with the simulated spectrum broken down into the major contributing species.

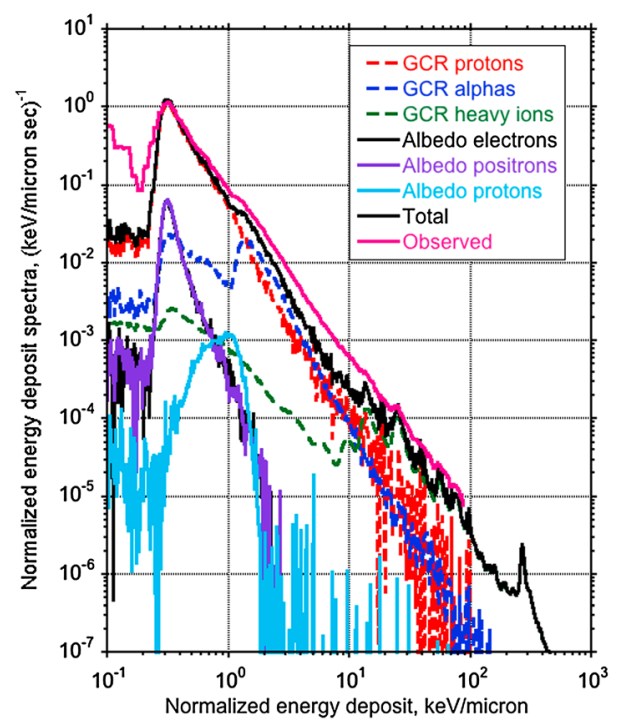

Figure 7. Energy deposit spectra in D6 of events triggering all three thick detectors, comparing the observed spectrum with the simulated spectrum broken down into the major contributing species.

albedo particles contribute only a few percent in either case. Statistical errors in the observations are very small on the scale of these plots, and deadtime correction is negligible during this period of solar quiet; the simulated curves in Figure 6 should be very smooth because of the 
broad angular acceptance of the single detector, so the statistical error for any given simulated species at any given flux level is shown by the magnitude of any "jaggedness" of those curves in Figure 6.

[26] The uncollimated response in Figure 6 includes particles coming in from all angles, so that the varying pathlengths of particles through the detector smear out any structure attributable to individual species. Agreement between simulation and observations as to shape is very good, and normalization is in agreement within a few tens of percent throughout the range between the $Z$ $=1$ minimum-ionizing peak at 0.3 kiloelectron-volts per micron and the saturation of the measurement at about 90 kiloelectron-volts per micron (below the minimumionizing peak, there is some contamination from thermal noise, which will make an insignificant error if integrated into a dose rate). The agreement between simulation and observations for triple coincidences in Figure 7 is good at the minimum-ionizing peak, but at higher energy deposits there are considerably more events in the observations than in the simulation, and they are spread more uniformly than in the simulation, which shows clearer peaks than in the observations for individual heavy GCR ions (with some jaggedness introduced by poor statistics at the extreme of the GCR proton curve, which is heavily weighted in the sum by the large number of primary protons producing these energy deposits). This is consistent with the comparison of Figures 2 and 5: the missing particles in the simulation in Figure 7 are from the more intense and smoother "wings" in observations like those of Figure 2, as compared to our simulations like those of Figure 5.

\section{Interpreting the Observations}

[27] As noted, interpretation of thick-detector measurements is complicated by events where a secondary particle completes a coincidence by remotely triggering a detector missed by a primary particle, as in the "wings" in Figures 2 and 5 . If we look at the subset of the data selected by requiring all six detectors to be triggered, the high thresholds of the thin detectors effectively reject these "wing" events, at the cost of also rejecting all proton and most helium events. At the larger energy deposits caused by heavier GCR nuclei, although, we find that our simulations and the observations agree very well as to their overall intensity, with some differences that shed light on the details of radiation transport and energy deposit as well as on the import of the heavy-ion nuclear reactions not yet included in our simulations.

[28] Figure 8 shows observations and simulations of the energy-deposit spectra in various detectors for these sixfold coincidence events. Because all these curves lie very nearly on top of one another, we have split them into four groups offset by factors of 10 on the vertical axis. The topmost two groups of three curves show observed (top) and simulated (second) energy-deposit spectra for the three thin detectors. One notes that our simulations are similar

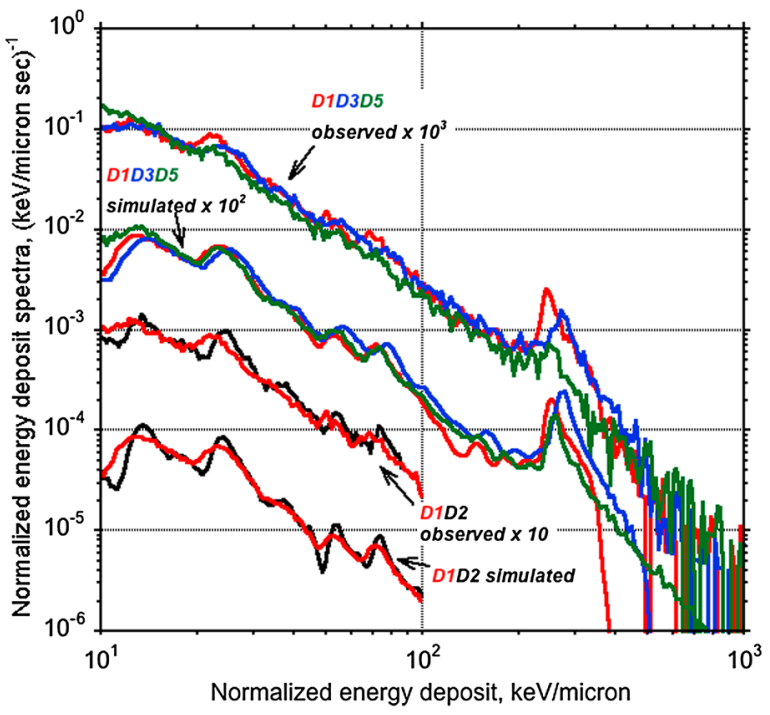

Figure 8. Comparison of simulated and observed energy-deposit spectra for events triggering all six detectors. Four groups of curves are presented, offset by one decade per set: from top to bottom are spectra for the three thin detectors (D1, D3, D5), observed; D1, D3, and D5, simulated; zenithward pair of detectors (D1 and D2), observed; and D1 and D2, simulated.

to the observations in one important way and dissimilar in another.

[29] First, in both sets of curves, the peaks due to individual species of relativistic GCR ions, notably iron around 250 kiloelectron-volts per micron, are shifted slightly toward higher energy deposits for D3 than for D1 or D5. This is the result of the loss of energy from the D1 and D5 detectors due to secondary particles escaping through the ends of the telescope. By contrast, D3 is surrounded by TEP both "upstream" and "downstream," and so any energy carried away from D3 by escaping particles will be replenished by particles generated nearby that travel to and deposit energy in D3. Thus, the energy-deposit spectrum for D3 would be more representative of what would be experienced by a sensitive target with shielding on all sides, or by a thick-enough target that the fractional energy escape from the target itself is minimal.

[30] The dissimilarity to be noted is that the individual GCR species' peaks, notably that for iron, become less pronounced in the observations as one moves from D1 to D3 to D5, while this effect is not seen in the simulations. This is because the "beam" of a given species of GCR ions entering D1 will lose progressively more primary particles to nuclear interactions as it travels deeper in the stack, an effect not yet included in our simulations. A more detailed analysis of this effect is presented by Zeitlin et al. (submitted manuscript, 2013).

[31] The bottom two curves in Figure 8 compare the observed (above) and simulated (bottom) energy-deposit spectra in the zenithward pair of detectors, D1 and D2. In both simulations and observations, we note that the peaks 
due to individual GCR heavy-ion species are both sharper and shifted somewhat to higher energy deposits for the thick D2 as compared to those for the thin D1. A particle's total energy deposit in the thick detector averages out energy-deposit fluctuations over a longer pathlength than in the thin detector, so that the distribution is more sharply clustered about the mean. That mean total energy deposit is also higher in the thick detector because secondary particles that might carry away energy stand a greater chance of being reabsorbed, and their energy added to the total, in the thicker detector than in the thinner.

[32] So the simulations give us confidence that the observations of six-detector coincidence events are well understood, with response almost entirely confined to the geometric acceptance cone defined by the detectors (no "wing" events), so that we can divide out the $0.569 \mathrm{~cm}^{2}$ sr geometric factor to abstract the measurement from the specifics of the sensor. But this data set rejects essentially all protons and almost all helium; to measure their contribution to energy deposit, our best data set is the set of events with a triple coincidence of the three thick detectors, which means we need to correct for particles arriving outside the acceptance cone. As a check on what a "clean" measurement of energy deposit would look like in the simulations, with no particles coming in outside the acceptance cone and minimal escape of secondary particles out the sides of the telescope (escape out the zenith and nadir ends is unavoidable), we simulated the response of the sensor to a "pencil" beam of GCR ions

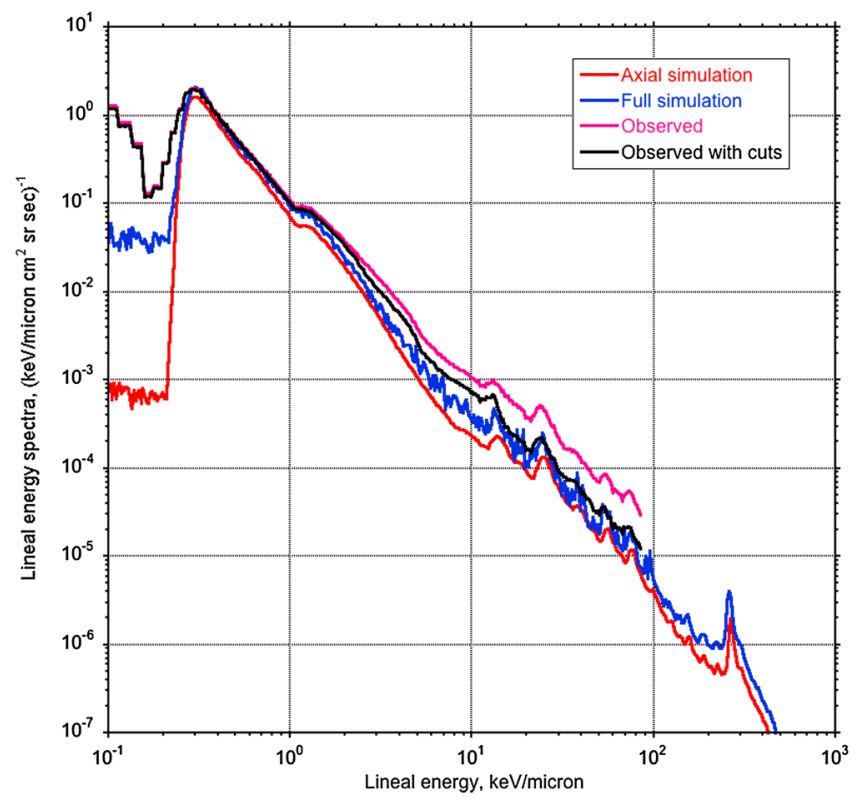

Figure 9. Comparison of energy-deposit spectra in D2 for events triggering all three thick detectors. Simulated spectra are shown both for GCRs coming in along the boresight axis and for the full environment, and observed spectra both without and with cuts to reduce the contribution from "wings" like those in Figures 2 and 5. (ignoring the small lunar albedo contribution) coming in parallel to the sensor axis and striking the center of D1.

[33] Figure 9 shows a comparison of the energy-deposit spectra in D2 for triple-coincidence events, with simulations of this "axial" illumination shown along with the full simulation results, and observations also shown both without and with a cut intended to reject the "wings." The observations and full simulation are divided by the $0.605 \mathrm{~cm}^{2} \mathrm{sr}$ geometric factor for triple coincidences (slightly wider than for sixfold because D1 is not included); the normalization of the axial simulation comes from the GCR flux per unit geometric factor. The energy deposit previously reported is converted to a lineal energy (see section 1) by correction for the difference between the detector thickness and the mean pathlength of events coming into the triple-coincidence acceptance cone, a matter of only a few percent.

[34] One can see that the iron peak in the axial simulation is shifted to a slightly higher energy deposit than that in the full simulation, because fewer secondary particles escape all the way from the sensor axis out the sides of the telescope. However, the main difference is that the full simulation includes many more events at larger energy deposits, due to particles coming in outside the nominal acceptance cone. The difference is significantly smaller in the region of the proton and helium peaks, below a few kiloelectron-volts per micron; this is not surprising, as ions with higher nuclear charge generate many more energetic secondary electrons, and therefore have a greater probability of seeing one travel far enough from an outof-aperture trajectory to make a "wing" coincidence having high energy deposits in the detector(s) actually hit by the ion. Thus a cut that rejects events with energy deposits closer to the plot axes than the proton and alpha tracks, avoiding the region below about 2 kiloelectron-volts per micron, should delete most of these events.

[35] Figure 9 shows the observed energy-deposit spectrum in D2 with no cuts, which is higher than the curve for the full simulation, as noted (for D6) in the discussion of Figure 7 above. Applying a cut as described to the observations, we see that this does indeed move the energy-deposit curve closer to that for the idealized axial simulation. This curve, labeled "Observed with cuts", may be taken as a preliminary measurement of the observed energy-deposit spectrum over the dynamic range of D2 (and subject to the energy constraint that the primary particles be energetic enough to penetrate the detector stack and reach D6, as discussed by Case et al. (submitted manuscript, 2013). We plan to refine these cuts if, as expected, we are better able to reproduce the observed distribution and intensity of the "wings" in a plot like Figure 2 by including nuclear interactions of heavy GCR ions in future simulations. In combination with observations of sixfold coincidence events at higher energy deposits as in Figure 8, then, CRaTER will let us assemble a good measurement of the lineal energy spectrum experienced by sensitive targets under shielding over a wide range of species and energy deposits, with 
confidence in the normalization per unit geometric factor as we reject particles arriving from outside the nominal acceptance cone.

\section{Appendix A: Altitude Dependence of Count Rates}

[36] At the Fall 2009 AGU meeting, early in the LRO mission, we presented some preliminary results from CRaTER, including an early version of Figure 2 [Spence et al., 2009]. Among the observations presented was a plot of the change in some detectors' countrates as LRO approached the Moon, similar to Figure A1. The curve labeled "Observed" is an average of the countrate of detector D1 as a function of altitude during the journey to the Moon and the initial establishment of orbit during days 171-179 of 2009. The curve labeled "Geometric" is what would be expected if the free-space countrate well away from the Moon declined in simple proportion to the fraction of the sky obscured by the disk of the Moon at each altitude. This is the profile that would be expected for a sensor isotropically sensitive to the GCR flux. Its normalization is arbitrary, and here it is matched up to the observations at about 1000 kilometers. Note that at lower altitudes the observed curve moves above the geometric curve; the effect is even more pronounced if the geometric curve is matched up to the observations using their average at higher altitudes. We proposed, at the time, that this might indicate a significant additional countrate coming from the Moon: a major albedo-particle source.

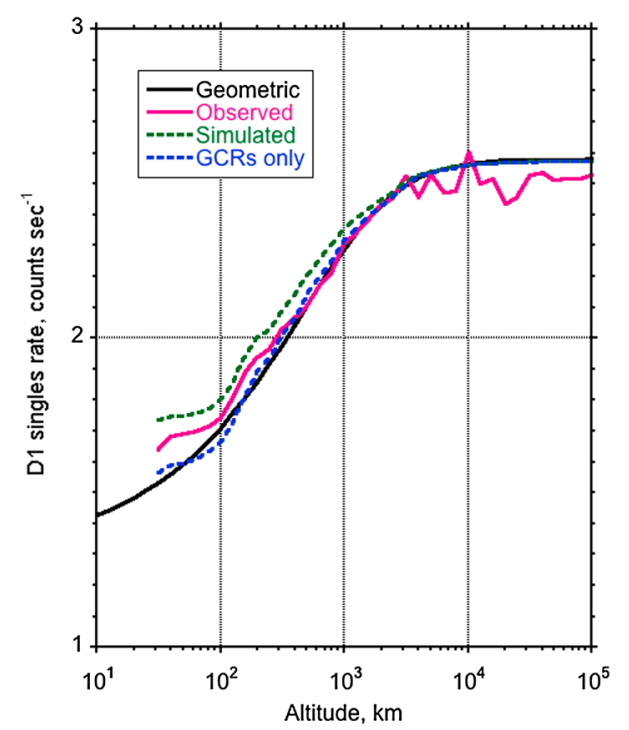

Figure A1. Observed and simulated D1 countrates vs. altitude during the journey of LRO to the Moon and into its initial orbits, along with a curve (with arbitrary normalization chosen to match up to the observations around 1000 kilometers) showing the simple ("geometric") expectation due to obscuration of an isotropic GCR flux by the Moon's disk.
[37] However, with detailed simulations of the response of the sensor and of the lunar secondary particles, we find that this is only partially true. Rather, much of the deviation from the geometric curve is a consequence of the nonisotropic response of D1 to GCRs, in particular to the protons that dominate the countrate. The broken curves labeled "Simulated" and "GCRs only" are the results of convolving the angular response of D1 to various species with the energy and angular distributions of GCRs and lunar albedo, summed up using the actual attitude and altitude of LRO during this period (the nominal zenith/nadir pointing had not been established at this time). There is a significant difference between the two simulated curves due to albedo particles, mostly protons. However, even the simulated curve without albedo contributions ("GCRs only") replicates the steeper drop-off with decreasing altitude around 200 kilometers, and the flattening of the curve below 100 kilometers, that are seen in the observations.

[38] The reason for this is that in the early orbits, between 35 and 200 kilometers altitude, LRO's sensors had a more zenith/nadir orientation at low altitudes and an orientation away from this at higher altitudes. Because of the high energy-deposit threshold of D1, it is not sensitive to protons coming straight in along the axis (from either direction); a proton at GCR energies must come in from well off axis to have a long enough pathlength in D1 to deposit enough energy to trigger it. When CRaTER is pointed nearly straight up and down, the Moon preferentially blocks protons coming in near the axis, to which D1 is not sensitive, so that its countrate decreases less than would be expected from the "Geometric" calculation. Conversely, when CRaTER is well

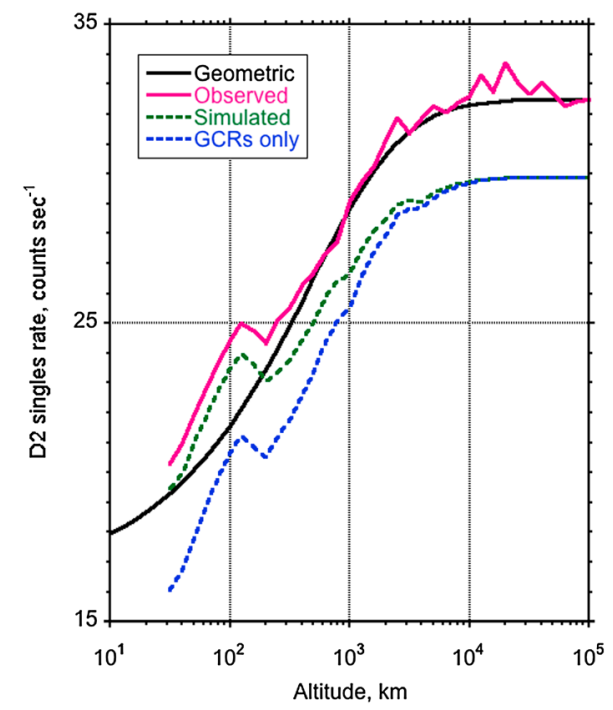

Figure A2. Observed and simulated D2 countrates, and expectation from simple obscuration geometry, as in figure A1. Peak in both observations and simulations around 200 kilometers is due to change in pattern of attitude vs. altitude when LRO was placed in an intermediate orbit with a periapse of 200 kilometers, but before beginning its nominal zenith-nadir oriented phase. 


\section{LOOPER ET AL.: LUNAR RADIATION ENVIRONMENT: CRATER}

away from this attitude, the Moon preferentially blocks particles to which D1 is sensitive, and its countrate drops off faster than expected. This is confirmed in observations and simulations (not shown) from days 181 to 257 of 2009, during which LRO was in a 35 to 200 kilometer orbit but with the zenith/nadir pointing enforced: during this period, the D1 countrate was noticeably higher than at these altitudes in the period of varying attitudes shown here.

[39] Further confirmation that much of the observed variation is an instrumental effect of nonisotropic sensitivity to GCRs is shown in Figure A2, which plots the same curves for D2. Unlike D1, the thick detector D2 is most sensitive to protons that come in parallel to the sensor axis, because off-axis shielding blocks lower-energy protons and, while it responds to all high-energy protons, its flat shape presents a larger projected target area (by a cosine factor) to particles coming in near the axis. Thus its countrate will be lower than the "Geometric" trend when the sensor is close to nominal zenith/nadir orientation, and vice versa when away from it. The increased fraction of time spent away from nominal orientation at high altitudes in the initial orbits, compared to the attitude at higher altitudes during approach to the Moon, results in the peak in both observed and simulated curves around 200 kilometers in Figure A2; this is purely an instrumental effect. Likewise, during days 181 to 257 (not shown), the zenith/nadir orientation resulted in countrates lower than plotted here at altitudes of 35 to 200 kilometers.

[40] Carrying this to a logical extreme, we can produce plots like these for the coincidence rates, whose response is strongly collimated along the sensor axis. In zenith/nadir orientation at low altitudes, the preferential blocking of GCRs arriving from directions to which these countrates are sensitive means that such countrates dropped toward $50 \%$ of their free-space values at altitudes much higher than expected from the "Geometric" curve, which on the face of it would suggest that the Moon was a sink larger than its geometric size for GCRs!
Therefore, while the simulations do show a significant contribution to these countrates from albedo particles (the difference between the "Simulated" and "GCRs only" curves), much of the variation that we initially called out as evidence for these particles turns out to have been an instrumental effect.

[41] Acknowledgments. We acknowledge the efforts of the CRaTER instrument team in developing and fielding the instrument hardware, as well as the LRO project team at NASA GSFC for the success of the LRO mission. This work was supported by NASA under contract GC189307NGA.

\section{References}

Allison, J., et al. (2006), Geant4 Developments and Applications, IEEE Trans. Nucl. Sci. 53 (1), 270-278, doi:10.1109/TNS.2006.869826.

Gasnault, O., et al. (2000), Lunar Fast Neutron Flux Calculation and Its Elemental Abundance Dependence, J. Geophys. Res. 105(E2), 4263-4271, doi:10.1029/1999JE001124.

ICRP, (1991), Quantities Used in Radiological Protection, Annex A of "ICRP Publication 60: 1990 Recommendations of the International Commission on Radiological Protection, Ann. ICRP 21(1-3), 79-89, doi:10.1016/0146-6453(91)90073-P.

Jia, Y., and Z. W. Lin, (2010), The Radiation Environment on the Moon from Galactic Cosmic Rays in a Lunar Habitat, Rad. Res. 173 (2), pp. 238-244, doi:10.1667/RR1846.1.

Mewaldt, R. A., et al. (2010), Record-Setting Cosmic-Ray Intensities in 2009 and 2010, Ap. J. Lett. 723, L1, doi:10.1088/2041-8205/723/1/L1.

Mrigakshi, A. I., et al. (2012), Assessment of Galactic Cosmic Ray Models, J. Geophys. Res. 117, A08109, doi:10.1029/2012JA017611.

O'Neill, P. M. (2010), Badhwar-O'Neill 2010 Galactic Cosmic Ray Flux Model - Revised, IEEE Trans. Nucl. Sci. 57 (6), pp. 3148-3153, doi:10.1109/TNS.2010.2083688.

Spence, H. E., et al. (2009), Early Results from the LRO Cosmic Ray Telescope for the Effects of Radiation (CRaTER) During This Historic Solar Minimum (Invited), Eos Trans. AGU 90 (52), Fall Meeting Supplement, Abstract U21C-07.

Spence, H. E., et al. (2010), CRaTER: The Cosmic Ray Telescope for the Effects of Radiation Experiment on the Lunar Reconnaissance Orbiter, Space Sci. Rev. 150, 243-284, doi:10.1007/s11214-009-9584-8.

Townsend, L. W., et al. (2011), Transmission of Galactic Cosmic Rays Through Mars Atmosphere, Space Weather 9, S00E11, doi:10.1029/ 2009 SW000564.

Wilson, J. K., et al. (2012), The First Cosmic Ray Albedo Proton Map of the Moon, J. Geophys. Res. 117, E00H23, doi:10.1029/2011JE003921. 